\title{
EFFECTS OF CUTTING PARAMETERS ON ACOUSTIC EMISSION SIGNAL RESPONSE DURING DRILLING OF LAMINATED COMPOSITES USING FACTORIAL DESIGN METHOD
}

\author{
P. Y. Andoh, F. Davis, and J. Antonio \\ Department of Mechanical Engineering, \\ Faculty of Mechanical and Agricultural Engineering, \\ Kwame Nkrumah University of Science and Technology, Kumasi.
}

\begin{abstract}
Factorial design has been used to study the effect of cutting parameters on acoustic emission signal response during the drilling of composite laminates. Experimental design is a strategy of planning, conducting, analyzing and interpreting experiments so that sound and valid conclusion can be drawn efficiently, and economically. In this study, effects of cutting speed, feed rate and tool diameter on the acoustic emission signal response are investigated using acoustic emission's energy, amplitude, root mean square and frequency responses and $2^{3}$ factorial design for drilling operation. Cutting tests were performed under dry conditions. Calculated effects, standard errors at $95 \%$ confidence level, and models governing the acoustic emission response to the cutting conditions have been generated from the acoustic emission signal responses. The results revealed that, acoustic energy response has significant effects due to the cutting parameters. Hence a model can be established to relate the acoustic emission's energy response and the cutting parameters, and as a result monitor and control the area of delamination during the drilling process.
\end{abstract}

Keywords: Composite Laminates, Machining, Acoustic Emission.

\section{INTRODUCTION}

Products made of composite materials usually undergo some form of intermediate processing such as drilling for assembly purposes. These processes could affect the structural integrity of the final component. These may be attributed to the selection of the cutting parameters such as cutting speed and feed rate for the drilling of the composite laminate. Although the damage area may be predicted through an analysis of the acoustic signals, the control action to reduce delamination is achieved through the cutting parameters. Practical issues in the drilling of composite materials include the proper selection of the material removal rate and the drill geometry to lower manufacturing cost and eliminate machining-induced defects. The main problems associated with the drilling of composite materials are delaminations in the panel, fiber pullout, and cracks, which lead to reduction of structural in- 
tegrity of the part (Hui, 1999). These flaws make machining-induced defects a suitable subject for further investigation. Damage mostly involves some formation of internal or surface cracks, voids, and delaminations. Damage in this work is defined as any change of state in the material, which alters the soundness of the structural integrity of the part. Considerable undetected material damage due to drilling, such as delaminations, could occur in composite materials before they are put into service. This may eventually lead to their shorter service life, the catastrophic failure of the product, and even the loss of human life (Ho-Cheng et al, 1990). Thus, investigation of the structural integrity of the composite during drilling is the major objective of this project. The drilling process is expected to affect the structural integrity of the part.

Non-destructive evaluation is an interdisciplinary field of study which is concerned with the development of analysis techniques and measurement technologies for the quantitative characterization of materials, tissues and structures by non-invasive means (Heyman, 1998). The common types of non-destructive evaluation techniques used to probe interior microstructures and to characterize subsurface features are acoustic emission, ultrasonic, radiographic, electromagnetic, optical scanning, electron microscopy, thermal imaging, liquid penetration inspection, magnetic particle and eddy current.

Non-destructive evaluation methods have been used in applications ranging from non-invasive medical diagnosis to on-line manufacturing process control, flaw detection, and materials characterization. The acoustic emission (AE) technique with its inherent in-process monitoring tool has several advantages over other nondestructive evaluation techniques and may become one of the most appropriate tools for process monitoring. This research seeks to develop a technique to control inter-ply delaminations during the drilling of laminated composites. A specific goal of this research is to study the effect of cutting parameter on the acoustic emission pa- rameters during drilling of carbon composite laminates.

\section{EXPERIMENTAL METHOD}

The material selected for the experiment was a 57 ply, 0.006 inch thick carbon/epoxy composite panel made of Amoco T300 3K Uni-Carbon in warp with S-glass in the fill direction. This material is referred to as "Amoco T300" in the rest of the work. A series of drilling operations were performed to study the effect of cutting parameters on the delamination area. In this work, AE tests were performed during each drilling operation. The schematic of the experimental set-up for the measurement of the AE signal and the drilling process is shown in Figure 1.

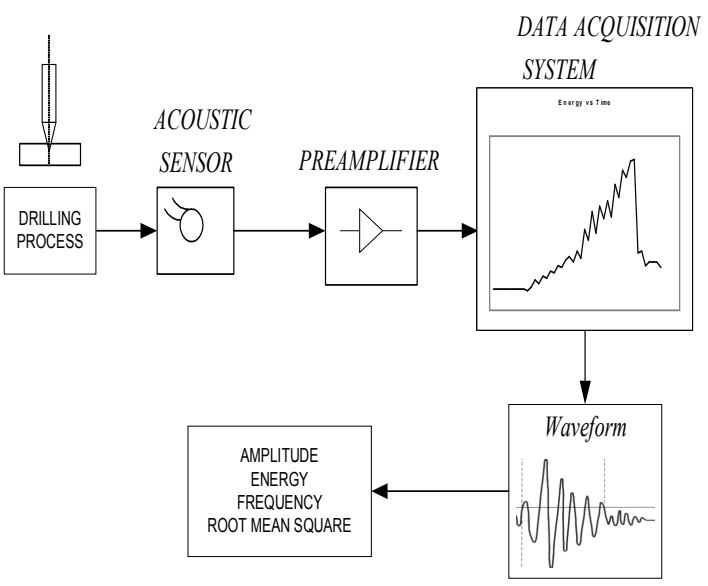

Figure 1: Schematic Showing Experimental Set-Up

The threshold frequency for the acoustic signal was set at $35 \mathrm{MHz}$ with a sample rate of $4 \mathrm{MHz}$, which is greater than ten times the natural frequency of the sensor used. This frequency was kept a good deal above the Nyquist range to avoid the aliasing problem. AE signal measurement parameters versus time plots were obtained for each drill test and the corresponding data were collected for subsequent analysis. An AE system consists of the sensor, cables, preamplifier, micro-processing and data storage systems.

A $150 \mathrm{kHz}$ sensor is coupled to the work piece with a fluid couplant (silicon grease) and is se- 
cured with tape. A preamplifier is placed close to the sensor to minimize electromagnetic interference. The preamplifier has a wide dynamic range and may drive the signal over a long length of cable so that the main instrumentation may be placed hundreds of meters from the test piece, if necessary. The preamplifier is inserted near the sensor for amplification of the signals. The preamplifier increases the signal strength by $40 \mathrm{~dB}$, with a band pass of $200 \mathrm{kHz}$. The choice of bandwidth in the preamplifier enables the elimination of spurious noise from the machinery and other mechanical processes unrelated to the acoustic emission test.

\section{FACTORIAL DESIGN EXPERIMENTAL RESULTS}

Factorial designs are of importance for a number of reasons: 1) They require relatively few runs per factor studied; 2) They can indicate major trends that determine promising directions for further experimentation. Three quantitative vari- ables, namely, feed rate $\left(\mathrm{f}-\mathrm{mms}^{-1}\right)$, cutting speed $(\mathrm{v}-\mathrm{mm} / \mathrm{min})$, and drill diameter $(\mathrm{d}-\mathrm{mm})$ are used to study the response of the AE signals. The amplitude $(\mathrm{A})$, energy (E), frequency (F) and root mean square values $(\mathrm{R})$ are monitored. Table 1 presents the levels of the variable used in this work. The cutting speed and the feed rate are selected to correspond to the typical range of values used in the drilling of composite laminates. The experiment is performed at each combination of the feed rate, cutting speed and tool diameter. Thus, there are $2^{3}=8$ experimental conditions.

For each setting, a hole is drilled and the acoustic signals are obtained. Each experiment is replicated at each point. The measurable quantities for the four responses are recorded. The averages of the two runs are computed for each set of conditions. Table 2 shows the experimental matrix and the magnitude of the amplitude response. Tables 3, 4 and 5 show the measurements for the energy, frequency, and root mean square values, respectively.

Table 1: The Experimental Matrix

\begin{tabular}{lcc}
\hline Variables & Lower Level (-) & Upper Level $(+)$ \\
\hline Feed Rate f $(\mathrm{mm} / \mathrm{sec})$ & 2 & 8 \\
Cutting Speed v $(\mathrm{mm} / \mathrm{min})$ & 1000 & 2500 \\
Tool Diameter d $(\mathrm{mm})$ & 6.4 & 12.8 \\
\hline
\end{tabular}

Table 2: The Measured Values for the Amplitude Response

\begin{tabular}{ccccccc}
\hline \multirow{2}{*}{ Pts } & & Code & \multicolumn{3}{c}{ Amplitude A ( dB) } \\
& f & v & d & Run 1 & Run 2 & Mean \\
\hline 1 & - & - & - & 65.00 & 84.00 & $\mathrm{~A}_{1}=74.50$ \\
2 & + & - & - & 100.00 & 100.00 & $\mathrm{~A}_{2}=100.00$ \\
3 & - & + & - & 74.00 & 72.00 & $\mathrm{~A}_{3}=73.00$ \\
4 & + & + & - & 89.00 & 96.00 & $\mathrm{~A}_{4}=92.50$ \\
5 & - & - & + & 83.00 & 75.00 & $\mathrm{~A}_{5}=79.00$ \\
6 & + & - & + & 88.00 & 93.00 & $\mathrm{~A}_{6}=90.50$ \\
7 & - & + & + & 82.00 & 99.00 & $\mathrm{~A}_{7}=90.50$ \\
8 & + & + & + & 86.00 & 96.00 & $\mathrm{~A}_{8}=91.00$ \\
\hline
\end{tabular}

Note: (-) represents the lower level of the variables,

(+) represents the upper level of the variables 
Table 3: The Measured Values for the Energy Response

\begin{tabular}{|c|c|c|c|c|c|c|}
\hline \multirow{2}{*}{ Pts } & \multicolumn{3}{|c|}{ Code } & \multicolumn{3}{|c|}{ Energy E $\left(v^{2} s\right)$} \\
\hline & f & $\mathbf{v}$ & d & Run 1 & Run 2 & Mean \\
\hline 1 & - & - & - & 7153 & 7163 & $E_{1}=7158.00$ \\
\hline 2 & + & - & - & 65535 & 65535 & $E_{2}=65535.00$ \\
\hline 3 & - & + & - & 14187 & 14716 & $\mathrm{E}_{3}=14451.50$ \\
\hline 4 & + & + & - & 36708 & 38061 & $E_{4}=37384.50$ \\
\hline 5 & - & - & + & 25572 & 25553 & $E_{5}=25562.50$ \\
\hline 6 & + & - & + & 53825 & 65535 & $E_{6}=59680.00$ \\
\hline 7 & - & + & + & 53247 & 55344 & $E_{7}=54295.50$ \\
\hline 8 & + & + & + & 60775 & 62651 & $\mathrm{E}_{8}=61713.00$ \\
\hline
\end{tabular}

Note: (-) represents the lower level of the variables, (+) represents the upper level of the variables

Table 4: The Measured Values for the Frequency Response

\begin{tabular}{ccccccc}
\hline Pts & & Code & & \multicolumn{3}{c}{ Frequency F (MHz) } \\
& f & v & d & Run 1 & Run 2 & Mean \\
\hline 1 & - & - & - & 130.00 & 123.00 & $\mathrm{~F}_{1}=126.50$ \\
2 & + & - & - & 165.00 & 146.00 & $\mathrm{~F}_{2}=155.50$ \\
3 & - & + & - & 120.00 & 136.00 & $\mathrm{~F}_{3}=128.00$ \\
4 & + & + & - & 138.00 & 130.00 & $\mathrm{~F}_{4}=134.00$ \\
5 & - & - & + & 152.00 & 166.00 & $\mathrm{~F}_{5}=159.00$ \\
6 & + & - & + & 141.00 & 146.00 & $\mathrm{~F}_{6}=143.50$ \\
7 & - & + & + & 144.00 & 136.00 & $\mathrm{~F}_{7}=140.00$ \\
8 & + & + & + & 163.00 & 174.00 & $\mathrm{~F}_{8}=168.50$ \\
\hline
\end{tabular}

Note: (-) represents the lower level of the variables,

(+) represents the upper level of the variables

Table 5: The Measured Values for the Root Mean Square Response

\begin{tabular}{ccccccc}
\hline \multirow{2}{*}{ Pts } & & Code & & \multicolumn{3}{c}{ Root Mean Square R (volts) } \\
& f & v & d & Run 1 & Run 2 & Mean \\
\hline 1 & - & - & - & 0.02 & 0.07 & $\mathrm{R}_{1}=0.05$ \\
2 & + & - & - & 1.34 & 0.21 & $\mathrm{R}_{2}=0.78$ \\
3 & - & + & - & 0.05 & 0.05 & $\mathrm{R}_{3}=0.05$ \\
4 & + & + & - & 0.12 & 0.34 & $\mathrm{R}_{4}=0.23$ \\
5 & - & - & + & 0.09 & 0.08 & $\mathrm{R}_{5}=0.09$ \\
6 & + & - & + & 0.19 & 0.45 & $\mathrm{R}_{6}=0.32$ \\
7 & - & + & + & 0.19 & 0.42 & $\mathrm{R}_{7}=0.31$ \\
8 & + & + & + & 0.21 & 0.37 & $\mathrm{R}_{8}=0.29$ \\
\hline
\end{tabular}

Note: (-) represents the lower level of the variables,

(+) represents the upper level of the variables 
The computed averages are used as the responses for each condition in calculating the main effects and the interaction effects for each $\mathrm{AE}$ response. The major goal of these experiments is to determine which of the responses are influenced by the cutting parameters and to generate a model that may be used to develop a control algorithm.

\section{ANALYSIS OF THE RESULTS}

The main effect of each of the process variables reflects the changes of the respective responses as the process variables change from a low to a high level. The average of the four measures is the main effect of the factor (variable) and is given as:

The main effect of the feed rate is

$$
E_{f}=\frac{1}{4}\left\{\begin{array}{l}
\left(A_{2}+A_{4}+A_{6}+A_{8}\right) \\
-\left(A_{1}+A_{3}+A_{5}+A_{7}\right)
\end{array}\right\}
$$

The main effect of the cutting speed is

$$
E_{v}=\frac{1}{4}\left\{\begin{array}{c}
\left(A_{3}+A_{4}+A_{7}+A_{8}\right) \\
-\left(A_{1}+A_{2}+A_{5}+A_{6}\right)
\end{array}\right\}
$$

The main effect of the drill size is

$$
E_{d}=\frac{1}{4}\left\{\begin{array}{l}
\left(A_{5}+A_{6}+A_{7}+A_{8}\right) \\
-\left(A_{1}+A_{2}+A_{3}+A_{4}\right)
\end{array}\right\}
$$

Two or more of the variables may jointly influence the responses. These joint influences are referred to as interactions. These interactions are given as follows:

The interaction between the feed rate and the cutting speed is defined as:

$$
I_{f v}=\frac{1}{4}\left\{\begin{array}{l}
\left(A_{1}+A_{4}+A_{5}+A_{8}\right) \\
-\left(A_{2}+A_{3}+A_{6}+A_{7}\right)
\end{array}\right\}
$$

The interaction between the feed rate and the drill diameter is defined as:

$$
I_{f d}=\frac{1}{4}\left\{\begin{array}{l}
\left(A_{1}+A_{3}+A_{6}+A_{8}\right) \\
-\left(A_{2}+A_{5}+A_{5}+A_{7}\right)
\end{array}\right\}
$$

The interaction between the cutting speed and the drill diameter is defined as:

$I_{v d}=\frac{1}{4}\left\{\begin{array}{l}\left(A_{1}+A_{2}+A_{7}+A_{8}\right) \\ -\left(A_{3}+A_{4}+A_{5}+A_{6}\right)\end{array}\right\}$

The three-factor interaction is expressed as

$I_{f v d}=\frac{1}{4}\left\{\begin{array}{l}\left(A_{2}+A_{3}+A_{5}+A_{8}\right) \\ -\left(A_{1}+A_{4}+A_{6}+A_{7}\right)\end{array}\right\}$

The mean of the runs is defined as

$$
E_{M}=\left[\sum_{1}^{8} A_{i} / 8\right]
$$

where $A_{i}$ are the acoustic parameters. The estimates for the four acoustic responses are shown in Table 6.

The results suggest that increasing the feed rate from 2 to $8 \mathrm{mms}^{-1}$ results in an increase of amplitude of the signal of $14.25 \mathrm{~dB}$; an increase of energy of $30711.25 \mathrm{v}^{2} \mathrm{~s}$; an increase of frequency of 12.00 MHz; and an increase of the root mean square value of 0.28 . Similarly, results also show that increasing the cutting speed from 1000 to $2500 \mathrm{~mm} / \mathrm{min}$ results in an increase of amplitude of the signal of $0.75 \mathrm{~dB}$; an increase of energy of $2477.25 \mathrm{v}^{2} \mathrm{~s}$; an increase of frequency of 3.50 $\mathrm{MHz}$; and an increase of the root mean square value of 0.09 . Also, the tool diameter increasing from 6.4 to $12.8 \mathrm{~mm}$ results in an increase of amplitude of the signal of $2.75 \mathrm{~dB}$, an increase of energy of $19180.50 \mathrm{v}^{2} \mathrm{~s}$; an increase of frequency of $16.75 \mathrm{MHz}$; and an increase of the root mean square value of 0.03 . These results may be confirmed by the application of the experimental error as discussed in the next section.

When genuine run replicates are created under a given set of experimental conditions, the variation among their associated observations are used to estimate the standard deviation of a single observation and, hence, the standard deviation of the results. In general, if $g$ sets of experimental condi- 
Table 6: Effects of Process Parameters on Acoustic Emission Signal Responses

\begin{tabular}{ccccc}
\hline $\begin{array}{c}\text { Effects/ } \\
\text { Interactions }\end{array}$ & Amp & Energy & Freq & RMS \\
\hline $\mathrm{E}_{\mathrm{M}}$ & 86.38 & 40722.50 & 144.38 & 0.26 \\
$\mathrm{E}_{\mathrm{f}}$ & 14.25 & 30711.25 & 12.00 & 0.28 \\
$\mathrm{E}_{\mathrm{v}}$ & 0.75 & 2477.25 & -3.50 & -0.09 \\
$\mathrm{E}_{\mathrm{d}}$ & 2.75 & 19180.50 & 16.75 & -0.03 \\
$\mathrm{I}_{\mathrm{fv}}$ & -4.25 & -15536.00 & 5.25 & -0.20 \\
$\mathrm{I}_{\mathrm{fd}}$ & -8.25 & -9943.75 & -5.50 & -0.17 \\
$\mathrm{I}_{\mathrm{vd}}$ & 5.25 & 12905.75 & 6.50 & 0.18 \\
$\mathrm{I}_{\mathrm{fvd}}$ & -1.25 & 2186.00 & 16.75 & 0.08 \\
\hline
\end{tabular}

tions are genuinely replicated and the $\mathrm{n}_{\mathrm{i}}$ replicate runs made at the $\mathrm{i}^{\text {th }}$ set yield an estimate $s_{i}^{2}$ having $\mathrm{v}_{\mathrm{i}}=\mathrm{n}_{\mathrm{i}}-1$ degree(s) of freedom (Hunter, 1978), the estimate of run variance is

$$
s^{2}=\frac{v_{1} s_{1}^{2}+v_{2} s_{2}^{2}+v_{3} s_{3}^{2}+\ldots+v_{g} s_{g}^{2}}{v_{1}+v_{2}+v_{3}+\ldots+v_{g}}
$$

With only $n_{i}=2$ replicates at each of the $g$ sets of conditions, the formula for the $i^{\text {th }}$ variance reduces to

$$
s_{i}^{2}=d_{i}^{2} / 2
$$

with $v_{i}=1$, where $d_{i}$ is the difference between the duplicate observations for the ith set of conditions. Thus, Equation 9 will yield

$$
s^{2}=\sum\left(d_{i}^{2} / 2\right) / g
$$

In general, if a total of $\mathrm{N}$ runs is made conducting a replicated factorial design, then the variance of an effect is given as

$V($ effect $)=\frac{4}{N} s^{2}$

and the standard error of the effect is given as

$$
s_{e}=\sqrt{V(\text { effect })}
$$

Using the results obtained for the AE responses during the drilling process and presented in Tables 2 to 5 , the $d_{i}$ and the $d_{i}^{2} / 2$ are computed for each $i^{\text {th }}$ condition. These values are used to compute the corresponding standard errors for each AE signal response. The results are presented in Table 7

However, it is not clear which of the estimates are important and which are unimportant. By examining the confidence intervals of each result, it can

Table 7: Standard Errors for the Acoustic Responses

\begin{tabular}{lcccc}
\hline \multicolumn{1}{c}{ Parameters } & Amp A (dB) & Energy E (v $\left.\mathbf{v}^{\mathbf{s}}\right)$ & Freq F (MHz) & RMS R (volts) \\
\hline Variance & 55.75 & $9.20 \mathrm{E}+06$ & 71.00 & 0.09 \\
Standard Error & 3.73 & $1.52 \mathrm{E}+03$ & 4.21 & 0.15 \\
\hline
\end{tabular}


Table 8: Summary of the Factorial Experimental Results

\begin{tabular}{|c|c|c|c|c|}
\hline \multirow{2}{*}{ Effects } & \multicolumn{4}{|c|}{ Responses } \\
\hline & Ampl., A (dB) & Energy, E $\left(v^{2} s\right)$ & Freq., F (MHz) & RMS, R (volts) \\
\hline $\mathrm{E}_{\mathrm{M}}$ & $86.37 \pm 3.73$ & $40722.5 \pm 1516.3$ & $144.37 \pm 4.21$ & $0.2625 \pm 0.15$ \\
\hline \multirow{2}{*}{$\mathrm{E}_{\mathrm{f}}$} & $14.25 \pm 3.73$ & $30711.25 \pm 1516.3$ & $12.00 \pm 4.21$ & $0.2825 \pm 0.15$ \\
\hline & Significant & Significant & Significant & Significant \\
\hline \multirow{2}{*}{$\mathrm{E}_{\mathrm{v}}$} & $0.75 \pm 3.73$ & $2477.25 \pm 1516.3$ & $(-) 3.50 \pm 4.21$ & $(-) 0.0875 \pm 0.15$ \\
\hline & Not Significant & Significant & Not Significant & Not Significant \\
\hline \multirow{2}{*}{$\mathrm{E}_{\mathrm{d}}$} & $2.75 \pm 3.73$ & $19180.5 \pm 1516.3$ & $16.75 \pm 4.21$ & $-0.025 \pm 0.15$ \\
\hline & Not Significant & Significant & Significant & Not Significant \\
\hline \multirow{2}{*}{$\mathrm{I}_{\mathrm{fv}}$} & $-4.25 \pm 3.73$ & $-15536 \pm 1516.3$ & $5.25 \pm 4.21$ & $-0.2 \pm 0.15$ \\
\hline & Significant & Significant & Significant & Not Significant \\
\hline \multirow{2}{*}{$\mathrm{I}_{\mathrm{fd}}$} & $-8.25 \pm 3.73$ & $-9943.75 \pm 1516.3$ & $-5.50 \pm 4.21$ & $-0.1725 \pm 0.15$ \\
\hline & Significant & Significant & Significant & Not Significant \\
\hline \multirow{2}{*}{$\mathrm{I}_{\mathrm{vd}}$} & $5.25 \pm 3.73$ & $12905.75 \pm 1516.3$ & $6.50 \pm 4.21$ & $0.1825 \pm 0.15$ \\
\hline & Significant & Significant & Significant & Not Significant \\
\hline \multirow{2}{*}{$\mathrm{I}_{\mathrm{fvd}}$} & $-1.25 \pm 3.73$ & $2186 \pm 1516.3$ & $16.75 \pm 4.21$ & $0.075 \pm 0.15$ \\
\hline & Not Significant & Significant & Significant & Not Significant \\
\hline
\end{tabular}

be determined if each effect or interaction is significant. These intervals are shown in Table 7. The combination of the results and the values in Tables 6 and 7 produce the final results for the factorial analysis. This is illustrated in Table 8 .

The significant effects and interactions are indicated in Table 8. A detail discussion of the results is presented in the next section.

\section{DISCUSSION}

The results of the factorial analysis are presented in Table 8 for all three AE signals parameters. Comparison of the estimates with the standard errors requires some interpretation. The main effect of a variable may be individually interpreted only if there is no evidence that the vari- able interacts with other variables. When there is evidence of one or more interactions, the interacting variables must be considered together.

Comparison of the amplitude response estimates with their standard errors (Table 8) and use of the normal probability limits suggests that the feed rate, (f), has an effect on the amplitude response. However, since the feed rate interacts with the drill diameter, $I_{f d}=-8.25 \pm 3.73$ ) suggests that the feed rate should not be treated individually. The two-way interaction between the drill diameter and feed rate is shown in Figure 2.

Figure 2 shows that increasing the tool diameter increases the amplitude at a low feed rate. However, increasing the tool diameter decreases the amplitude at a high feed rate. Increasing the feed

104 Journal of Science and Technology, Vol. 27, No. 3, December 2007 


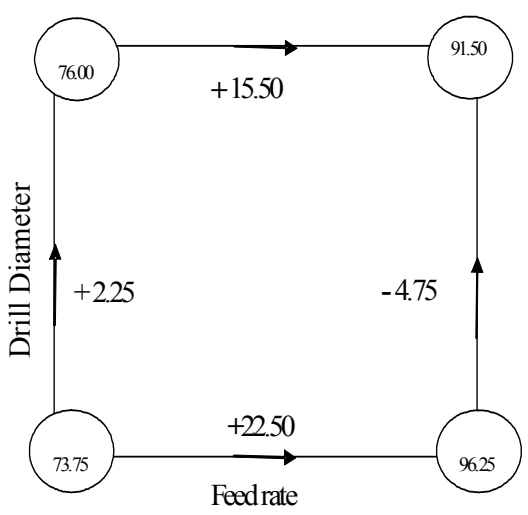

Fig. 2: The Amplitude Response for the Two-Way Interaction between Feed Rate and Drill Diameter

rate increases the amplitude, irrespective of the tool diameter. In addition, the cutting speed interacts with the drill diameter $\left(I_{v d}=5.25 \pm 3.73\right)$. Increasing the tool diameter decreases the amplitude at a low cutting speed. However, the amplitude increases at a higher cutting speed. Increasing the cutting speed decreases the amplitude at a small tool diameter. However, the amplitude increases with a larger tool diameter, resulting in an increase in the cutting speed.

The normal probability limits and the comparison of the root mean square response estimates with their standard errors suggest that there are no interaction effects among the three cutting parameters. The root mean square value depends mainly on the feed rate.

A comparison of the estimates with their standard errors, and the normal probability limits show that the feed rate (f) and the drill diameter (d) have effects on the energy response. However, since the feed rate interacts with both the speed and the drill diameter $\left(I_{f v}=\right.$ $-15536 \pm 1516.33$ and $I_{f d}=-9943.75 \pm 1516.33$, respectively) and the drill diameter interacts with the speed $\left(I_{v d}=12905 \pm 1516.33\right)$, the feed rate and drill diameter should not be treated separately. The two-way interaction for the cutting speed and feed rate is shown in Figure 3.

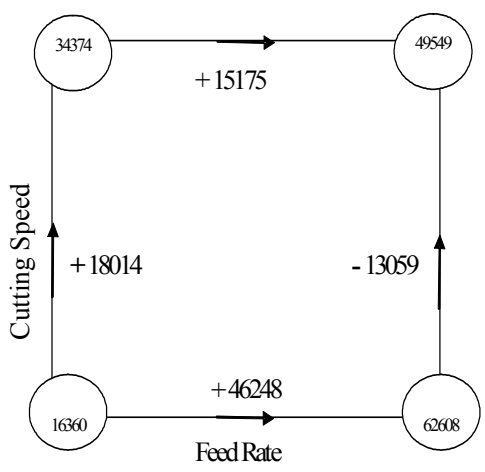

Fig. 3: The Energy Response for the Two-Way Interaction between Feed Rate and Cutting Speed

Figure 3 shows that increasing the cutting speed, increases the energy at a low feed rate. However, the energy decreases at a higher feed rate. Increasing the feed rate increases the energy, irrespective of the cutting speed. It is also known that increasing the cutting speed decreases the energy at a small tool diameter. However, the energy increases at a larger tool diameter. The energy increases as the tool diameter increases, irrespective of the cutting speed. However, the energy increases with the tool size at low feed rates while it decreases at high feed rates. In addition, the energy increases with an increasing feed rate, irrespective of the tool diameter.

A comparison of the frequency response estimates with their standard errors, and the normal probability plot suggest that the feed rate and the drill diameter have effects on the frequency response. However, the feed rate interacts with both the speed and the drill diameter $\left(I_{f v}=5.25 \pm 14.21\right.$ and $\left.I_{f d}=-5.50 \pm 4.21\right)$. The two-way interaction between the drill diameter and the feed rate is shown in Figure 4.

Figure 4 shows that both the feed rate and the drill diameter are increased, while the frequency also increases, irrespective of the feed rate and the drill diameter. The frequency decreases as the cutting speed increases at low feed rates. How- 


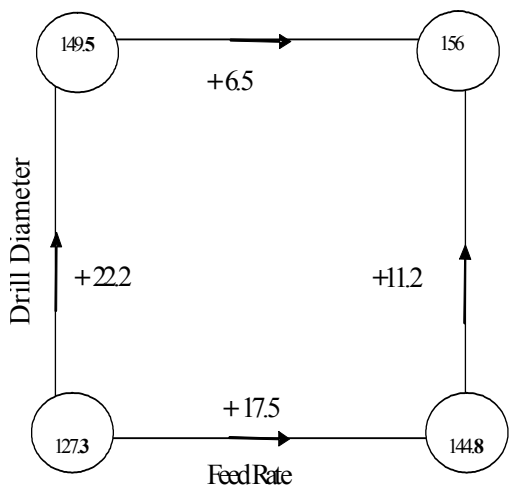

Fig. 4: The Frequency Response for the Two-Way Interaction between Feed Rate and Drill Diameter

ever, increasing the cutting speed increases the frequency at high feed rates. Similarly, the frequency increases with an increasing feed rate, irrespective of the cutting speed. In addition, the drill diameter interacts with the cutting speed $\left(I_{v d}\right.$ $=6.50 \pm 4.21$ ). Increasing the tool diameter decreases the frequency at a low cutting speed. However, the frequency increases at higher cutting speeds. The frequency increases as the cutting speed increases, irrespective of the tool diameter.

There is evidence of three-factor interactions among the three cutting parameters for the energy and frequency responses $\left(I_{f v d}=\right.$ $2186 \pm 1516.33$ for energy and $I_{f d}=16.75 \pm 4.21$ for frequency). Thus, these three variables must be considered together. An interpretation of the results under such conditions is very difficult in the deterministic approach. Other approaches, such as the stochastic approach or modeling, may be used for further analysis and interpretation of these results. The discussion so far suggests that models of the $\mathrm{AE}$ signal parameters and the cutting parameters may be established and used to develop an algorithm for monitoring, forecasting and control of the size of delamination during the drilling process.

\section{CONCLUSION}

A study of the effects of cutting conditions on acoustic emission signals response have been conducted using statistical factorial design method. The significant effects were obtained for each acoustic emission parameter. From the results, it can be concluded that the energy response has the highest significant effects due to the cutting parameters. Hence a model can then be established to relate the acoustic emission energy response and the cutting parameters which can be used to monitor and control the area of delamination during the drilling process.

\section{REFERENCES}

Andoh, P. Y, (2005), "Monitoring And Control Of Delamination in the Drilling of Carbon/ Epoxy Composite Laminates", PhD Dissertation, North Carolina A \& T State University, Greensboro.

Andoh, P. Y and Owusu Ofori, S. P., (2004), "A Quantitative Parameter for Non-Destructive Evaluation of Drilled Laminated Composite Parts", Transaction of North America Manufacturing Research Institution of SME, Charlotte, pp. 335-342.

Heyman, J., (1998), "NDE of Damage in Thick Stitched Composites Using Laser Based Ultrasound with Optical Fibers for Remote Generation and Detection", Materials Evaluation, 56 (12), 1402 - 1411.

Ho-Cheng, H., and Dharan, C. K. H., (1990), "Delamination during Drilling in Composite Laminates", ASME Journal of Engineering for Industry, pp. 39-47.

Hui, D., (1999), "Sixth Annual International Conference On Composites Engineering ICCE/6", Orlando, Florida, pp. 213-227.

Hunter, W. G. Box, G. E. P., and Hunter, J. S., (1978), Statistic for Experimenters: An introduction to Design, Data Analysis, and Model Building, Wiley, pp. 306-344. 